\title{
A Common Exocytotic Mechanism Mediates Axonal and Dendritic Outgrowth
}

\author{
Sonia Martinez-Arca, ${ }^{1,4}$ Silvia Coco, ${ }^{2}$ Gaëll Mainguy, ${ }^{3}$ Ursula Schenk, ${ }^{2}$ Philipp Alberts, ${ }^{1,4}$ Pascale Bouillé, ${ }^{5}$ \\ Mauro Mezzina, ${ }^{5}$ Alain Prochiantz, ${ }^{3}$ Michela Matteoli, ${ }^{2}$ Daniel Louvard, ${ }^{4}$ and Thierry Galli 1,4 \\ ${ }^{1}$ Membrane Traffic and Neuronal Plasticity, Institut National de la Santé et de la Recherche Médicale U536, Institut du Fer- \\ à-Moulin, F-75005 Paris, France, ${ }^{2}$ Synaptic Development and Function, Cellular and Molecular Pharmacology and Bruno \\ Ceccarelli Centers, Consiglio Nazionale delle Ricerche, 20129 Milan, Italy, ${ }^{3}$ Developmental and Cellular Neurobiology, \\ Centre National de la Recherche Scientifique (CNRS) Unité Mixte de Recherche (UMR) 8542, Ecole Normale Supérieure, \\ F-75005 Paris, France, ${ }^{4}$ Morphogenesis and Cell Signaling, CNRS UMR 144, Institut Curie, F-75005 Paris, France, and \\ 5Gene Therapy, Genethon III, Unité de Recherche Associée 1923, CNRS, BP 60, F-91002 Evry Cedex, France
}

Outgrowth of the dendrites and the axon is the basis of the establishment of the neuronal shape, and it requires addition of new membrane to both growing processes. It is not yet clear whether one or two exocytotic pathways are responsible for the respective outgrowth of axons and dendrites. We have previously shown that tetanus neurotoxin-insensitive vesicleassociated membrane protein (TI-VAMP) defines a novel network of tubulovesicular structures present both at the leading edge of elongating dendrites and axons of immature hippocampal neurons developing in primary culture and that TI-VAMP is an essential protein for neurite outgrowth in PC12 cells. Here we show that the expression of the $\mathrm{N}$-terminal domain of TI-VAMP inhibits the outgrowth of both dendrites and axons in neurons in primary culture. This effect is more prominent at the earliest stages of the development of neurons in vitro. Expression of the N-terminal domain deleted form of TI-VAMP has the opposite effect. This constitutively active form of TI-VAMP localizes as the endogenous protein, particularly concentrating at the leading edge of growing axons. Our results suggest that a common exocytotic mechanism that relies on TI-VAMP mediates both axonal and dendritic outgrowth in developing neurons.

Key words: axonal outgrowth; dendritic outgrowth; exocytosis; SNARE; TI-VAMP; adeno-associated virus; neurons in primary culture
Growth of an axon and extension of a dendritic tree are the key morphological features of neuronal development, and they define neuronal shape (Prochiantz, 1995). Exocytosis plays a fundamental role in axonal and dendritic outgrowths because both processes involve major increases in the surface area of the plasma membrane (Futerman and Banker, 1996; Bradke and Dotti, 1997). This new surface area is added at the tips of the elongating processes (Craig et al., 1995; Zakharenko and Popov, 1998). The axon and the dendrites differ in their contents of protein early in neuronal development and are controlled by different factors (Prochiantz, 1995). Nevertheless, when the axon of a neuron is severed, a new axon forms from a pre-existing dendrite (Dotti and Banker, 1987; Bradke and Dotti, 2000a). The molecular machinery controlling the trafficking of membranes involved in axonal

Received Dec. 22, 2000; revised March 9, 2001; accepted March 19, 2001.

This work was supported in part by Action Concertée Incitative-Jeunes Chercheurs (Grant 5254) from the Ministère de la Recherche et des Technologies to T.G and by Telethon Italia (Grant 1042) and European Economic Community (Grant BIO4-CT98-0408) to M.M. S.M.A. is a recipient of a fellowship from Fondation pour la Recherche Médicale and P.A. from the Deutscher Akademischer Austauschdienst. We thank the Production Service Unit of Genethon III for large-scale AAV vector production in the frame of the Gene Vector Production Network (GVPN; www.genethon.fr/gvpn) program. The GVPN is supported by the Association Française contre les Myopathies (Evry, France). We are gratef ul to Jean-Antoine Girault for his constant support and for critical reading of this manuscript. We thank Jack Kyte for helpful comments and corrections and R. Jahn and W. Hong for the generous gift of antibodies.

S.M., S.C., and G.M. contributed equally to this work.

Correspondence should be addressed to Dr. Thierry Galli, "Membrane Traffic and Neuronal Plasticity," CNRS UMR144, Institut Curie, Section de Recherche, 26 rue d'Ulm, 75231 Paris Cédex 05, France. E-mail: thierry.galli@curie.fr.

Copyright (C) 2001 Society for Neuroscience $0270-6474 / 01 / 213830-09 \$ 15.00 / 0$ and dendritic outgrowth is still poorly characterized, and it is not yet clear whether one or two independent exocytotic pathways are responsible for the respective outgrowth of axons and dendrites (Bradke and Dotti, 2000b).

Soluble $N$-ethylmaleimide-sensitive fusion protein (NSF) attachment protein (SNAP) receptors (SNAREs) are important proteins of membrane traffic because they are the core molecules of membrane fusion (Söllner et al., 1993; Bock and Scheller, 1999; Jahn and Sudhof, 1999). In neurons, the plasma membrane target SNAREs (t-SNAREs), syntaxin 1 and SNAP25, are found all along the plasma membranes of axons and dendrites (Garcia et al., 1995; Galli et al., 1995). Both the outgrowth of axons and the outgrowth of dendrites depend on SNAP25 because they are inhibited by the botulinum neurotoxins $\mathrm{A}$ and $\mathrm{C} 1$ but do not involve synaptobrevin 2 because they are not affected by tetanus neurotoxin (Osen-Sand et al., 1996; Igarashi et al., 1996; Grosse et al., 1999), which cleaves synaptobrevin 2 . The recent discovery of the clostridial neurotoxin-resistant vesicular SNARE tetanus neurotoxin-insensitive vesicle-associated membrane protein (TIVAMP) (Galli et al., 1998) (also called VAMP-7; Advani et al., 1998), the product of the Synaptobrevin-like gene 1 (D'Esposito et al., 1996) in neuronal cells (Coco et al., 1999; Martinez-Arca et al., 2000a,b) has provided an appealing hypothesis for the observed lack of effect of tetanus neurotoxin on axonal and dendritic outgrowth (Johannes and Galli, 1998). An important structural feature of TI-VAMP is its N-terminal extension of 100 amino acids (Galli et al., 1998). We have shown that this domain inhibits the formation of SNARE complexes both in vivo and in 
vitro. In agreement with this observation, we observed that neurite outgrowth is inhibited by expression in pheochromocytoma 12 (PC12) cells of the $\mathrm{N}$ terminus of TI-VAMP and enhanced by expression of a form of TI-VAMP deleted at its $\mathrm{N}$ terminus (Martinez-Arca et al., 2000a). Each process from a differentiated PC12 cell contains both dendritic and axonal markers, and none make synaptic contacts so these cells cannot be considered to be a true model of neuronal differentiation and maturation. In this paper, we expressed the $\mathrm{N}$-terminal domain and the form of TI-VAMP deleted at its $\mathrm{N}$ terminus in neurons to assay for the role of TI-VAMP in the outgrowth of axons and dendrites.

\section{MATERIALS AND METHODS}

Antibodies and clones. A polyclonal antibody directed against green fluorescent protein (GFP) was generated in rabbit and affinity-purified over recombinant glutathione $S$-transferase-coupled GFP. Mouse monoclonal antibodies directed against GFP (clones 7.1 and 13.1; Roche Diagnostics, GmbH, Germany), the Golgi matrix protein of $130 \mathrm{kDa}$ (GM130; Transduction Laboratories, Lexington, KY) and syntaxin 6 (clone 30; Transduction Laboratories) have been described previously. Rabbit polyclonal antibody directed against calreticulin was from Affinity Bioreagents. Mouse monoclonal antibody directed against synaptobrevin 2 (clone 69.1) and rabbit polyclonal antibody directed against syntaxin 7 were generous gifts of Drs. R. Jahn (Max Planck Institute, Goettingen, Germany) and W. Hong (Institute of Molecular and Cell Biology, Singapore), respectively. Antibodies against excitatory amino acid carrier 1 (EAAC1) and GluR1 were kind gifts of Drs. Rothstein (Johns Hopkins University, Baltimore, MD) and Wenthold (Uniformed Services University of the Health Sciences, Bethesda, MD), respectively, and were used as previously described (Coco et al., 1997). The cDNA of human TIVAMP and the GFP-fusion constructs have been previously described (Galli et al., 1998; Martinez-Arca et al., 2000a).

Electroporation of mouse embryonic brain. Brains were dissected out from mouse embryonic day 13 (E13) embryos in PBS and $0.6 \%$ glucose. Plasmids $(2 \mu \mathrm{g} / \mu \mathrm{l})$ were co-injected with $0.05 \%$ Fast-Green (Sigma, St. Louis, MO) in telencephalic vesicles using a glass capillary needle. Electroporation was performed essentially as described in Miyasaka et al. (1999), by five pulses $(50 \mathrm{~V}, 50 \mathrm{msec})$ with a T-820 apparatus using a 10-mm-diameter tweezertrode electrode (BTX, San Diego, CA). After electroporation cells were dissociated in trypsin $0.05 \%$ (Life Technologies, Cergy-Pontoise, France), plated on glass coverslips coated with matrigel (Collaborative Biomedical Products, Bedford, MA), and cultured as in Mainguy et al. (2000). After the indicated times, cells were fixed with 4\% PFA and either mounted with Vectashield-4',6-diamidino2-phenylindole (DAPI) (Vector Laboratories, Burlingame, CA) for observation of direct GFP-signal or permeabilized with $0.3 \%$ Triton X-100 and processed for immunofluorescence as described (Coco et al., 1999).

Adeno-associated virus vectors construction, production, purification, and titration. Recombinant adeno-associated virus (rAAV)-cytomegalovirus (CMV)-GFP-TI-VAMP, rAAV-CMV-GFP-Nter-TI-VAMP, and rAAV-CMV-GFP- $\triangle$ Nter-TI-VAMP vectors were respectively obtained from the pCR3.1 GFP-TI-VAMP, pCR3.1 GFP-Nter-TI-VAMP, pCR3.1 GFP- $\triangle$ Nter-TI-VAMP, plasmids, harboring the corresponding transgene and the pGG2 AAV plasmid. The latter plasmid is derived from the pSUB201 plasmid, in which the expression is driven by hMV promoter and stabilized by the SV40 late polyA and a chimeric intron composed of the $5^{\prime}$ donor splice site of the first intron of the human $\beta$ globin gene (hBB) and the $3^{\prime}$ acceptor splice site of the intron of an Ig gene (IgG) heavy chain variable region. First, GFP-TI-VAMP, GFPNter-TI-VAMP, and GFP- $\triangle$ Nter-TI-VAMP sequences were PCRamplified by using specific primers and the high-fidelity pfu turbo polymerase (Stratagene, La Jolla, CA) and further digested by NheI restriction enzyme at the $3^{\prime}$ end. These fragments were purified from agarose gel by using the Geneclean kit (BIO101, Vista, CA) according to the manufacturer's procedure. Second, the pGG2 plasmid was cut by NheI and EcoRV enzymes to add the PCR-amplified cDNAs. The correct orientation of the inserted sequences were checked by DNA sequencing analysis and agarose gel electrophoresis. Large-scale production and purification of vectors were performed by using the triple transfection of 293 cells, followed by $\mathrm{CsCl}$ density gradients purification, as previously described (Xiao et al., 1998). The infectious particle concentration is determined by a variation of the procedure previously described (Salvetti et al., 1998).
Adenoassociated viral infection of neurons. Cortical and striatal neurons were prepared from rat E16 embryos as described previously (Rousselet et al., 1990). After dissociation, neurons were plated in collagen-coated glass coverslips in chemically defined medium as above. Five hours after plating cells were infected overnight with the described Aavs at a multiplicity of infection of 100 in a final volume of $50 \mu l$. The day after, the Aavs were removed and cells were kept in regular medium for the indicated periods of time. The direct GFP signal from the Aav-encoded proteins could be detected $3 \mathrm{~d}$ after infection, however, because of the low level of expression of the transgenes and to facilitate detection of the infected neurons for subsequent quantitation, cells were fixed and permeabilized as described above and stained with anti-GFP antibodies.

Transfection of hippocampal neurons with calcium phosphate. Calcium phosphate crystals were prepared as described in Maniatis et al. (1982). For transfection, neurons were placed in medium conditioned by cortical astrocytes for at least $15 \mathrm{hr}$. Calcium phosphate crystals were left for $4 \mathrm{hr}$, and the cells were then washed accurately with Krebs'-Ringer's solution and transferred in their previous medium.

Quantification of axonal and dendritic length in hippocampal neurons. Randomly chosen fields were taken with a Bio-Rad MRC-1024 Confocal Microscope equipped with a LaserSharp 3.2 software. Acquired images were processed and quantitatively analyzed with NIH Image 1.62 software from National Institute of Health, resulting in the analysis of between 40 and 60 GFP-positive cells, for each condition and for each independent experiment. Multiple dendrites emerging from a same stalk were counted as distinct entities. For immunocytochemistry, neurons were permeabilized with $0.3 \%$ Triton X-100 and processed for immunofluorescence as described (Coco et al., 1999).

Quantification of axonal length in corticostriatal neurons. Randomly chosen fields were taken with a MicroMax CCD camera (Princeton Instruments), resulting in the analysis of between 10 and 50 (in the electroporation experiments) or between 25 and 200 (in the Aav experiments) GFP-positive cells, for each condition and for each independent experiment. Quantification of axonal length was done using the Metamorph software (Princeton Instruments). Double immunofluorescence with neuronal markers was performed to verify exclusively quantification of neuronal cells. The obtained data were analyzed for their statistical significance with Sigma Stat (SPSS, Inc.).

Quantification of Nter-TI-VAMP-induced cell death. After electroporation of embryonic E13 brains, cells were dissociated and cultured in the absence or presence of $200 \mu \mathrm{M}$ zVAD (Calbiochem, La Jolla, CA) to inhibit caspases thus apoptosis. After $24 \mathrm{hr}$ all the green fluorescent neuronal and non-neuronal cells remaining were scored.

\section{RESULTS}

\section{Expression of the $\mathrm{N}$-terminal domain of TI-VAMP inhibits neuronal differentiation}

To investigate the role of TI-VAMP in neuronal differentiation, we expressed GFP and GFP fused to the N-terminal domain of TI-VAMP (GFP-Nter-TI-VAMP), using a calcium phosphatebased transfection method, in E18 rat hippocampal neurons that were cultured in the presence of feeding glial cells (Fig. 1). Neurons were transfected at $1 \mathrm{~d}$ in vitro (div) or $4 \mathrm{div}$ and examined $24 \mathrm{hr}$ later. We observed that neurons expressing GFPNter-TI-VAMP grew shorter neurites, whereas those expressing GFP differentiated as nontransfected neurons (Fig. 1) (data not shown). We quantitated the growth of axons and dendrites, these processes being defined by morphological criteria (Dotti et al., 1988). The inhibition affected both processes. Interestingly, dendrites of neurons expressing GFP-Nter-TI-VAMP were fewer in number and shorter than those of neurons expressing GFP, after 2 and 5 div; the strongest effect was after 2 div (Fig. 1B,C). In comparison, the inhibitory effect of the expression of GFP-NterTI-VAMP on axonal outgrowth appeared stronger than the effect on dendritic outgrowth, when only the lengths of the processes were compared (Fig. 1, compare $B, D$ ).

We confirmed that dendritic function was significantly altered in hippocampal neurons expressing GFP-Nter-TI-VAMP because a reduced localization of the glutamate transporter EAAC1 

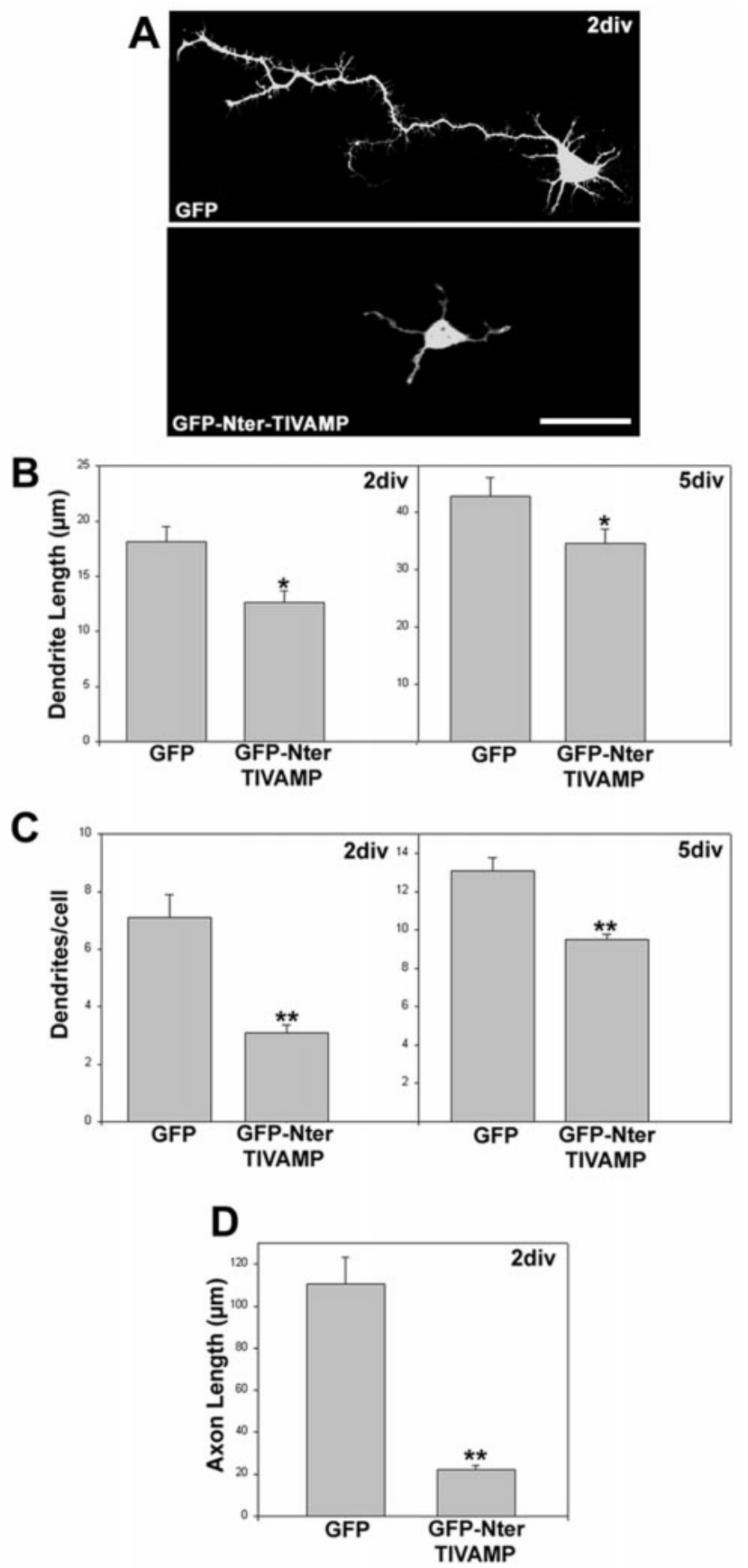

Figure 1. Expression of the N-terminal domain of TI-VAMP inhibits axonal and dendritic growth. $A$, Hippocampal neurons from E18 rats transfected with GFP or GFP-Nter-TI-VAMP $4 \mathrm{hr}$ after plating and fixed $24 \mathrm{hr}$ later. Scale bar, $32 \mu \mathrm{m}$. $B$, Cells transfected as in $A$ after 1 or 4 div were recorded $24 \mathrm{hr}$ later, and the dendritic length was measured. $C$, Cells transfected as in $A$ after 1 or 4 div were recorded $24 \mathrm{hr}$ after transfection, and the number of dendrites on each cell was counted. $D$, Cells were transfected as in $A$ after 1 div with GFP or GFP-Nter-TI-VAMP, and the axonal length was measured $24 \mathrm{hr}$ later; shown are the mean values $\left( \pm\right.$ SEM) of between 40 and 60 analyzed cells. ${ }^{* *} p<0.006 ;{ }^{*} p<0.06$.

was seen in the dendrites of these cells compared with cells expressing only GFP (Fig. 2). EAAC1 is a transmembrane protein expressed in dendrites of hippocampal neurons at very early stages of their development, and thus is a good marker of vesicular transport to the growing dendritic plasma membrane (Coco et al., 1997). Quantification of dendrites positive for EAAC1
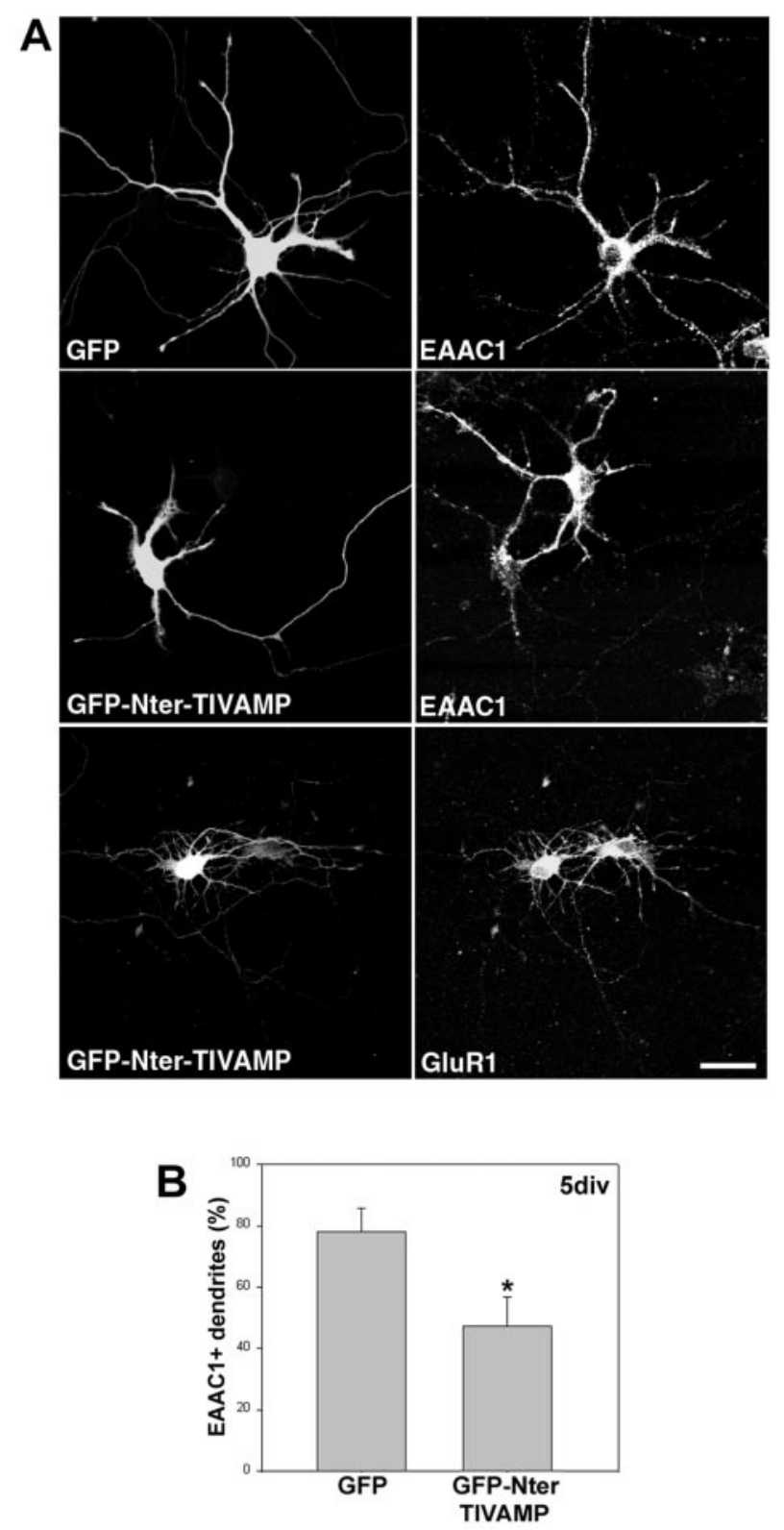

Figure 2. Expression of the N-terminal domain of TI-VAMP affects the distribution of EAAC1 but not GluR1 in hippocampal neurons. $A$, Fourday-old hippocampal neurons from E18 rats were transfected with GFP or GFP-Nter-TI-VAMP, and after $24 \mathrm{hr}$ they were fixed and stained for the indicated proteins. Note the expected dendritic localization of EAAC1 in control neurons and in neurons transfected with GFP (right top panel and nontransfected cell in the right middle panel) compared with its general lower expression and specifically its absence from the dendrites in cells expressing Nter-TI-VAMP (transfected cell in the right middle panel). By contrast, the level of expression and the localization of GluR1 were not affected by expression of GFP-Nter-TI-VAMP (compare the two cells in the right bottom panel). Scale bar, $21 \mu \mathrm{m}$. B, Cells transfected as in $A$ were stained $24 \mathrm{hr}$ later for EAAC1; shown are the mean values ( \pm SEM) of percentage of GFP- or Nter-TI-VAMP-positive dendrites labeled also for EAAC1.

showed that far fewer of them were from neurons expressing GFP-Nter-TI-VAMP than from neurons expressing only GFP (Fig. $2 B$ ). We did not observe any effect on the localization of the AMPA receptor subunit GluR1 (Fig. 2A). This lack of effect shows that only a subset of transport pathways are inhibited by the expression of GFP-Nter-TI-VAMP. 
A
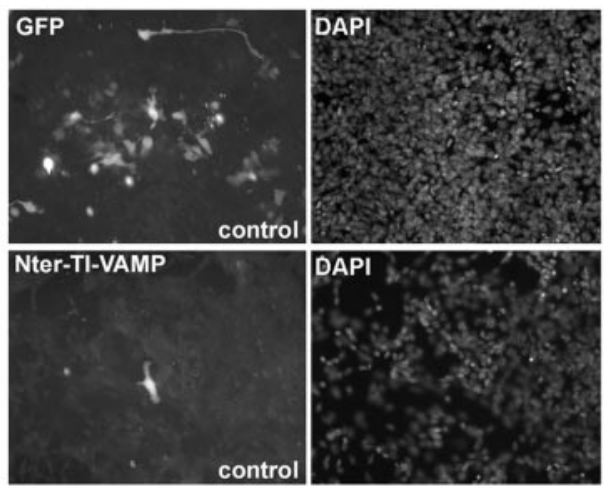

B

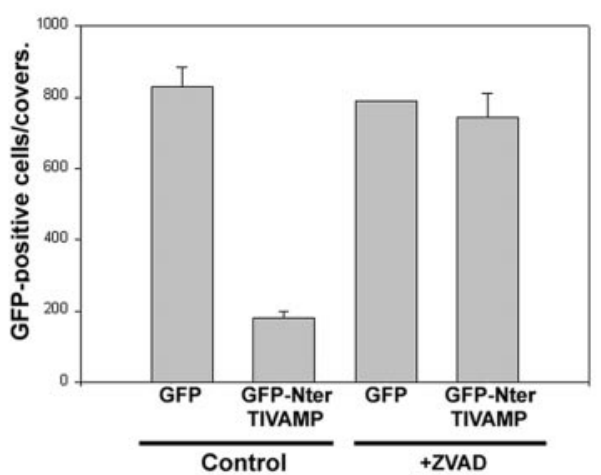

D
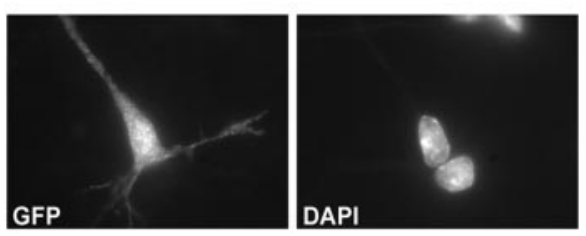
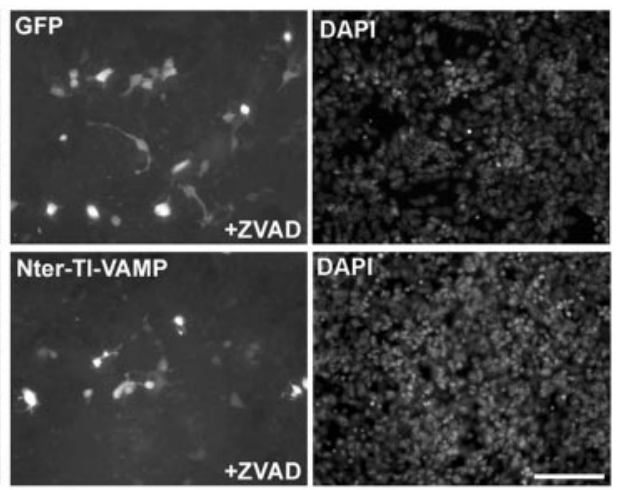

C
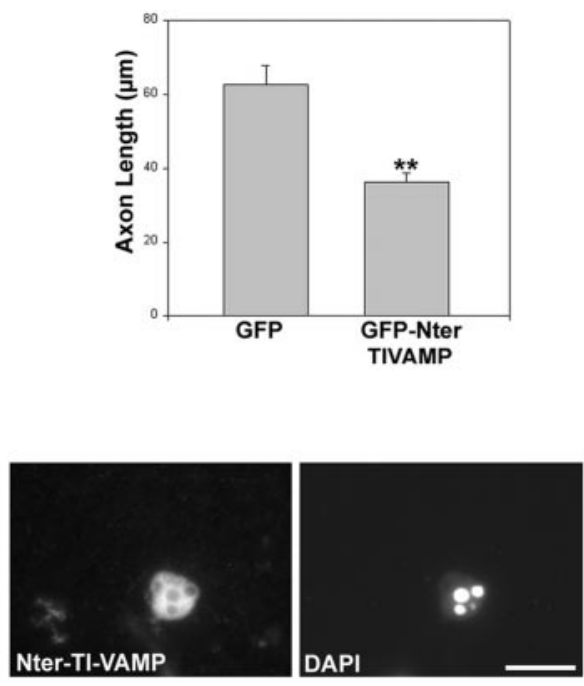

Figure 3. Expression of the N-terminal domain of TI-VAMP induces apoptosis. $A$, Corticostriatal neurons from intact embryonic brains were electroporated with the indicated constructs and cultured for $24 \mathrm{hr}$ in the absence (left panels) or presence (right panels) of the caspase inhibitor zVAD. Observe the increase in the number of transfected cells in zVAD-treated Nter-TIVAMP-electroporated cells compared with nontreated cells. In the case of GFPelectroporated cells, there is no difference between zVAD-treated or nontreated cells. Scale bar, $100 \mu \mathrm{m}$. B, Quantification of the apoptotic effect of the $\mathrm{N}$-terminal domain of TI-VAMP in cells treated as in $A$; shown are the mean values $( \pm$ SEM $)$ of the number of positive cells on each coverslip. $C$, Quantification of the effect in axonal length of the expression of the N-terminal domain of TIVAMP in cells treated as in $A$. Shown are the mean values $( \pm$ SEM) of a minimum of 40 cells. $D$, Neurons infected with Aav carrying GFP or Aav carrying GFP-Nter-TIVAMP fixed $3 \mathrm{~d}$ after infection. A representative cell of each type is shown. Note that the cell expressing GFP displays neurites and a normal nucleus compared with a noninfected cell, whereas the cell expressing Nter-TI-VAMP is round, with no neurites and presents a typical apoptotic nucleus as seen with DAPI staining. Scale bar, $20 \mu \mathrm{m}$.
Our observation that the inhibitory effect of GFP-Nter-TIVAMP was smaller when the transfection was performed in 4 div hippocampal neurons compared with 1 div neurons prompted us to study axonal and dendritic outgrowth in embryonic neurons of earlier stages. For doing so, we electroporated intact embryonic brains from E13 mice, a stage of brain development in which neuroblasts are very abundant. Then, we dissociated the cortices and striata and cultured neurons and astrocytes. The cells were plated, cultured, and observed after 1-3 div. Using this approach, cells expressing GFP were abundant at both time points. The development of neurons expressing GFP was indistinguishable from that of nontransfected neurons, and normal axonal and dendritic outgrowth was observed. However, after 1 div, we could find only few cells expressing GFP-Nter-TI-VAMP and after 3 div, almost none were visible (Fig. $3 A$ ). Presumably, expression of GFP-Nter-TI-VAMP at early times during development blocked neuronal differentiation and rapidly induced neuronal cell death. To test this presumption, we treated the cells with benzyloxycarbonyl-Val-Ala-Asp-(OMe)-fluoromethylketone (zVAD), a broad-spectrum inhibitor of caspases (Polverino and Patterson, 1997), shortly after plating. Cells treated with zVAD and expressing GFP-Nter-TI-VAMP survived after 1 div but differentiation, as assessed by axonal and dendritic outgrowth, was severely impaired when compared with the corresponding cells expressing GFP alone (Fig. 3A). Quantification of survival showed that zVAD reversed the pro-apoptotic effect induced by the expres- sion of GFP-Nter-TI-VAMP (Fig. 3B). The neurons treated with zVAD and expressing GFP-Nter-TI-VAMP surviving after 1 div showed a significant reduction in axonal length relative to cells expressing GFP alone (35 $\mu \mathrm{m}$ compared with $60 \mu \mathrm{m})$ (Fig. $3 C$ ), and only $16 \%$ of their axons were $>50 \mu \mathrm{m}$ compared with $62 \%$ in the case of GFP. This result suggests that the deleterious effect of the expression of Nter-TI-VAMP was attributable, at least in part, to inhibition of axonal and dendritic outgrowth. The electroporation of intact brain, however, led to a high level of expression of the transgenes, and the apoptotic effect could be caused by nonspecific toxicity at high intracellular concentration, although the control cells seemed normal. Therefore, we constructed recombinant Aavs (Du et al., 1996; Slack and Miller, 1996) expressing GFP or GFP-Nter-TI-VAMP and used them to infect corticostriatal neurons. In this case also, the expression of GFP-NterTI-VAMP resulted in strong inhibition of axonal and dendritic outgrowths, after 1, 2, and 3 div (Figs. 3D, 4). Staining with DAPI showed that the nuclei of cells expressing GFP-Nter-TI-VAMP, but not the nuclei of cells expressing GFP, was condensed and fragmented. This effect was seen already in some GFP-Nter-TIVAMP-expressing neurons after 1 div (data not shown) but affected virtually all of the cells after 3 div (representative cells are depicted in Fig. 3D). Thus, the expression of GFP-Nter-TIVAMP, mediated by the corresponding recombinant Aav, resulted in neuronal cell death also, in spite of the fact that infection with Aav induced a much lower level of expression than electro- 

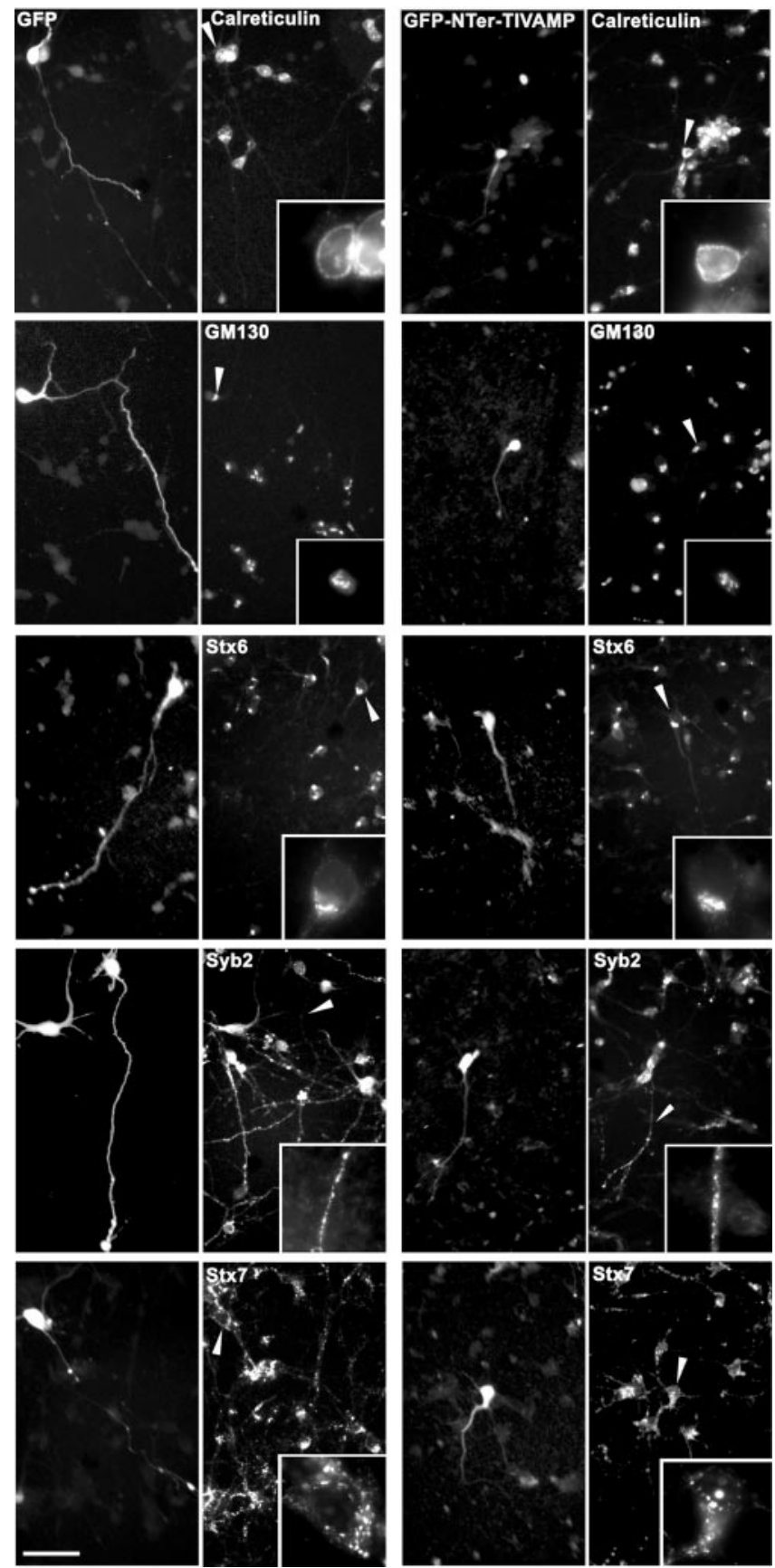

Figure 4. Cells expressing the N-terminal domain of TI-VAMP show normal secretory and endocytic pathways. Corticostriatal neurons from E16 rats were infected with Aav carrying GFP or Aav carrying GFP-NterTI-VAMP, fixed $1 \mathrm{~d}$ after infection, and double-labeled for GFP and the indicated markers of the secretory (calreticulin, GM130, syntaxin 6, synaptobrevin 2) and endocytic (syntaxin 7) pathways. At this time, some Nter-TI-VAMP-expressing cells are not yet apoptotic, although they already present shorter neurites compared with GFP-expressing cells. Note, however, that the different markers localized similarly in GFP- and GFP-Nter-TI-VAMP-expressing neurons. Insets show higher magnifications of the areas indicated by the arrowheads. GM130, Golgi matrix protein of $130 \mathrm{kDa}$; Stx6, syntaxin 6; Syb2, synaptobrevin 2; Stx7, syntaxin 7. Scale bar, $60 \mu \mathrm{m}(15 \mu \mathrm{m}$ in insets $)$.

poration. Indeed, the transfected peptides were detected by immunofluorescence using an anti-GFP antibody in the case of viral expression, whereas they could be seen by direct GFP-emitted fluorescence in the electroporation experiments. Consequently, this effect was independent of the method of transfection. Furthermore, to discard the possibility that overexpression of GFPNter-TI-VAMP could have pleiotropic effects and inhibit several fusion events, we analyzed both the secretory and endocytic pathways in cells expressing GFP or GFP-Nter-TI-VAMP. Figure 4 shows representative neurons expressing GFP or GFP-Nter-TIVAMP. We conducted this study short times after infection (1 d) to look at the cells expressing GFP-Nter-TI-VAMP before they became apoptotic. We found no difference in the distribution of calreticulin, a marker of the endoplasmic reticulum (ER) (Patil et al., 2000), GM130, a protein of Golgi stacks (Nakamura et al., 1997), syntaxin 6, a SNARE of the trans-Golgi network (Bock et al., 1997), synaptobrevin 2, and syntaxin 7, an endosomal SNARE (Wong et al., 1998), in cells expressing GFP-Nter-TI-VAMP compared with those expressing GFP or to noninfected cells (Fig. 4). Altogether, these results demonstrated that TI-VAMP is one of the proteins essential for both axonal and dendritic outgrowth.

\section{A constitutively active form of TI-VAMP enhances axon outgrowth}

We have previously found that the expression of a form of TI-VAMP from which the N-terminal domain has been deleted (GFP- $\Delta$ Nter-TI-VAMP) stimulated neurite outgrowth in PC12 cells (Martinez-Arca et al., 2000a). Because expression of GFPNter-TI-VAMP blocked both dendritic and axonal outgrowth in neurons, we asked whether expression of GFP- $\Delta$ Nter-TI-VAMP had any effect on these processes. Transfection of plasmids expressing GFP- $\Delta$ Nter-TI-VAMP by electroporation of intact E13 murine brains greatly stimulated axonal outgrowth of neurons in primary culture compared with GFP (Fig. 5A,B). Rat corticostriatal neurons infected with Aavs producing expression of GFP$\triangle$ Nter-TI-VAMP also showed increased axonal outgrowth compared with GFP (Fig. 5A,C). These stimulatory effects could be seen at $1,2,3$, or 6 div, but the strongest effects were observed after 3 and 6 div in the case of Aav-treated neurons. Most remarkably, expression of GFP- $\Delta$ Nter-TI-VAMP increased fourfold the percentage of axons $>300 \mu \mathrm{m}$ after 6 div (Fig. $5 C$ ). The expression of full-length TI-VAMP had no effect relative to the expression of GFP (Fig. 5). We did not observe any significant effect of the expression of GFP- $\Delta$ Nter-TI-VAMP on dendritic length or on the number of dendrites per cell in any of the two models we have used (our unpublished observation).

Removal of the N-terminal extension of TI-VAMP produces a molecule of protein that has a structure typical of synaptobrevin 2. It was possible that GFP- $\Delta$ Nter-TI-VAMP has lost important targeting information and behaves as synaptobrevin 2 because it does not reach its site of normal function. If this were the case, these results would not provide insight into the function of TI-VAMP in axonal and dendritic outgrowth. Therefore, we studied the subcellular location of GFP- $\Delta$ Nter-TI-VAMP and synaptobrevin 2 in corticostriatal neurons 6 div after infection with Aavs. GFP- $\Delta$ Nter-TI-VAMP was found in cell bodies, dendrites, axon hillocks, all along the axon, and in varicosities (Fig. $6)$. We found that GFP- $\Delta$ Nter-TI-VAMP did not colocalize with synaptobrevin 2 . Interestingly, GFP- $\Delta$ Nter-TI-VAMP densely localized at the leading edge of axons in the peripheral region of growth cones, a location devoid of synaptobrevin 2 (Fig. 6E), as seen for the endogenous protein (Coco et al., 1999).

\section{DISCUSSION}

Our previous work has shown that TI-VAMP is one of the proteins essential for neurite outgrowth in PC12 cells (Martinez- 

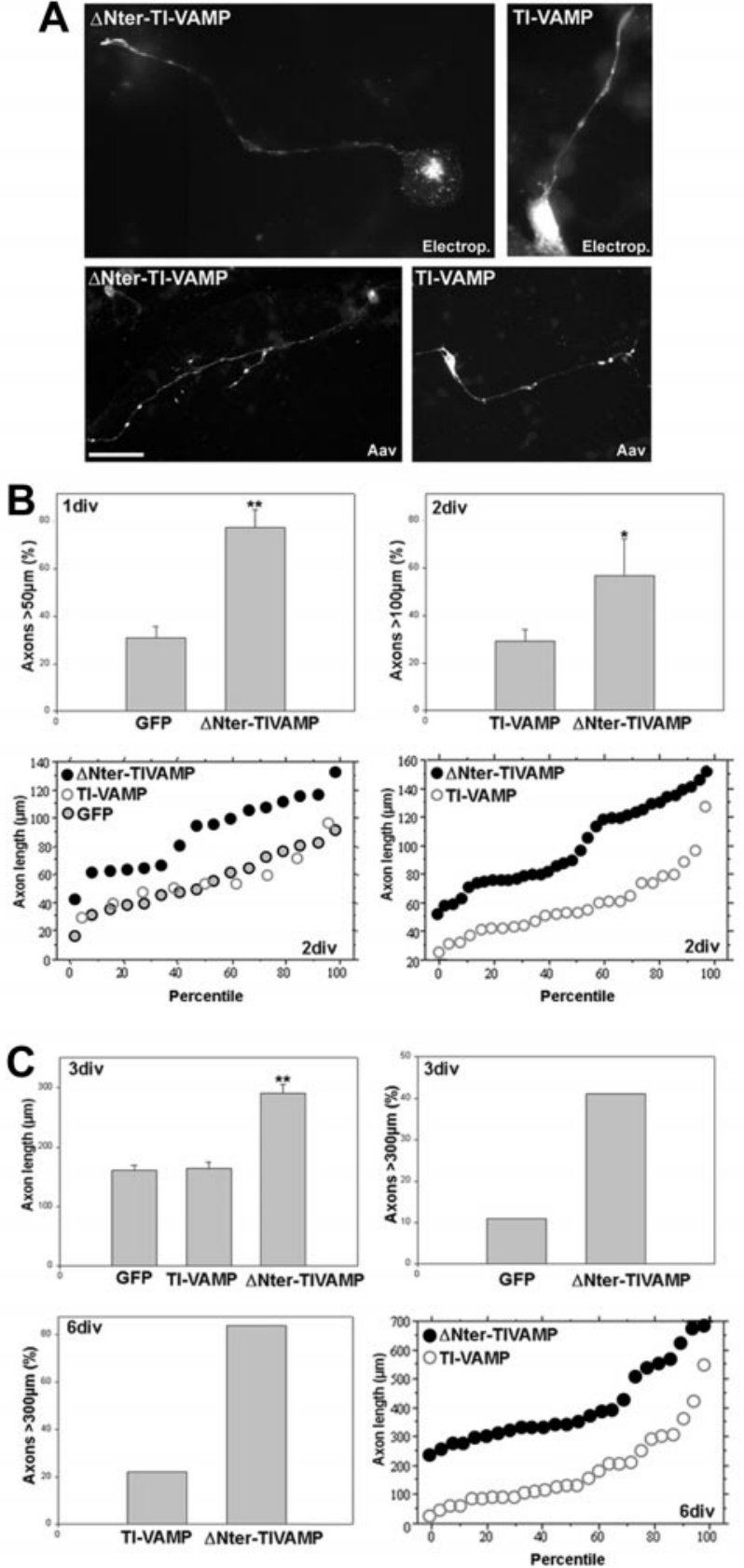

Figure 5. Expression of $\Delta$ Nter-TI-VAMP activates axonal growth. $A$, Intact brains from E13 mice (top panels) or corticostriatal neurons from E16 rats (bottom panels) were electroporated or infected with the indicated Aavs, respectively. Cells in primary culture were fixed after 2 (electroporation) or $3 \mathrm{div}$ (Aavs). Note the punctate distribution in the cell body and along the axon of both full-length GFP-TI-VAMP and GFP- $\Delta$ Nter-TI-VAMP and the fact that GFP- $\Delta$ Nter-TI-VAMPexpressing cells present longer axons than cells expressing GFP-TIVAMP. Scale bar: $20 \mu \mathrm{m}$ (top panels); $60 \mu \mathrm{m}$ (bottom panels). B, Quantification of the effect in axonal growth of the expression of $\Delta$ Nter-TIVAMP in electroporated neurons. Neurons expressing GFP, GFP-TIVAMP, or GFP- $\triangle$ Nter-TI-VAMP were fixed after the indicated times, and the length of their axons was measured. In the top panels the mean values $( \pm$ SEM) of percentage of axons longer than 50 or $100 \mu \mathrm{m}$ are shown from three independent experiments; the bottom panels show two representative experiments. $C$, Quantification of the effect on axonal growth of the expression of $\Delta$ Nter-TI-VAMP in Aav-infected neurons. Neurons expressing the indicated constructs were fixed after 3 or 6 div, and their axonal length was measured; each panel shows a representative experiment. ${ }^{*} p<0.001 ;{ }^{*} p<0.005$.
Arca et al., 2000a). We have shown that TI-VAMP concentrates at the leading edge of axonal and dendritic outgrowths of immature hippocampal neurons (Coco et al., 1999). Here, we provide evidence that TI-VAMP is essential for both dendritic and axonal outgrowth in neurons. Expression of the N-terminal domain of TI-VAMP inhibited axonal and dendritic outgrowth. Expression of a form of TI-VAMP from which the N-terminal domain has been deleted strongly enhanced axonal outgrowth in mouse cortical and striatal neurons but had no effect on dendritic outgrowth. The fact that the expression of these two proteins had opposite effects on the outgrowth of axons shows that the observed changes were not the result of the transfection itself but the functional property of the proteins themselves.

In this study, we were not able to characterize the biochemical effects of Nter-TI-VAMP and $\triangle$ Nter-TI-VAMP, because such studies in transfected neurons are technically limited by the small number of transfected cells. Nevertheless, we have previously found that the N-terminal domain of TI-VAMP inhibits the capacity of this v-SNARE to form complexes with syntaxin 1 and SNAP25, its target Q-SNAREs in PC12 cells (Martinez-Arca et al., 2000a). TI-VAMP presents a long N-terminal extension that is not present in members of the synaptobrevin family of R-SNAREs (Galli et al., 1998). Preliminary results show that a chimera consisting in the fusion of the N-terminal domain of TI-VAMP with synaptobrevin 2 leads to a v-SNARE with a reduced capacity to form complexes with syntaxin 4 and SNAP23 in fibroblasts (S. Martinez-Arca and T. Galli, unpublished results). Altogether, our results suggest a model in which TI-VAMP would be a less active but a more controlled v-SNARE than synaptobrevin 2 . The N-terminal domain of TI-VAMP is unlikely to contain targeting signals because the localization of $\Delta$ Nter-TIVAMP is similar to that of the full-length protein (Figs. 5, 6) (Coco et al., 1999). Moreover, the stimulatory effect on axonal outgrowth resulting from expression of $\Delta$ Nter-TI-VAMP (Fig. 5) is likely to be specific for TI-VAMP because $\Delta$ Nter-TI-VAMP does not colocalize with synaptobrevin 2 (Fig. 6) despite the fact that it has a similar structure and high primary sequence similarity (Galli et al., 1998). Given the facts that TI-VAMP concentrates at the tip of axonal and dendritic outgrowths (Coco et al., 1999) and forms SNARE complexes with plasma membrane syntaxin 1 and SNAP25 (Martinez-Arca et al., 2000a), that $\triangle$ Nter-TI-VAMP forms more abundant SNARE complexes with syntaxin 1 and SNAP25 (Martinez-Arca et al., 2000a), and that its expression in PC12 cells (Martinez-Arca et al., 2000a) and neurons (this study) accelerates neurite outgrowth, it seems reasonable to propose that TI-VAMP mediates an important exocytotic mechanism involved in neuronal differentiation.

Expression of Nter-TI-VAMP inhibited neuronal differentiation (Fig. 1) and led to neuronal cell death (Fig. 3). This effect cannot be attributable to a general deleterious effect of this peptide because both the secretory and the endocytic pathway are normal in cells before they enter the apoptosis (Fig. 4). Expression of Nter-TI-VAMP did not affect smooth ER generation and transport, intra- and post-Golgi trafficking, and the formation and transport of synaptic vesicle and endocytic fusion events. Moreover, the expression of axonal and dendritic cytoskeletal components such as tau and MAP-2 is not altered (data not shown), and we have previously shown that Nter-TI-VAMP inhibits neurite outgrowth but does not lead to cell death in PC12 cells (MartinezArca et al., 2000a). It was recently shown that the Shc site of TrkB controls both neuronal survival and axonal outgrowth by activating the PI3-kinase and MEK signaling pathways, thus establishing 

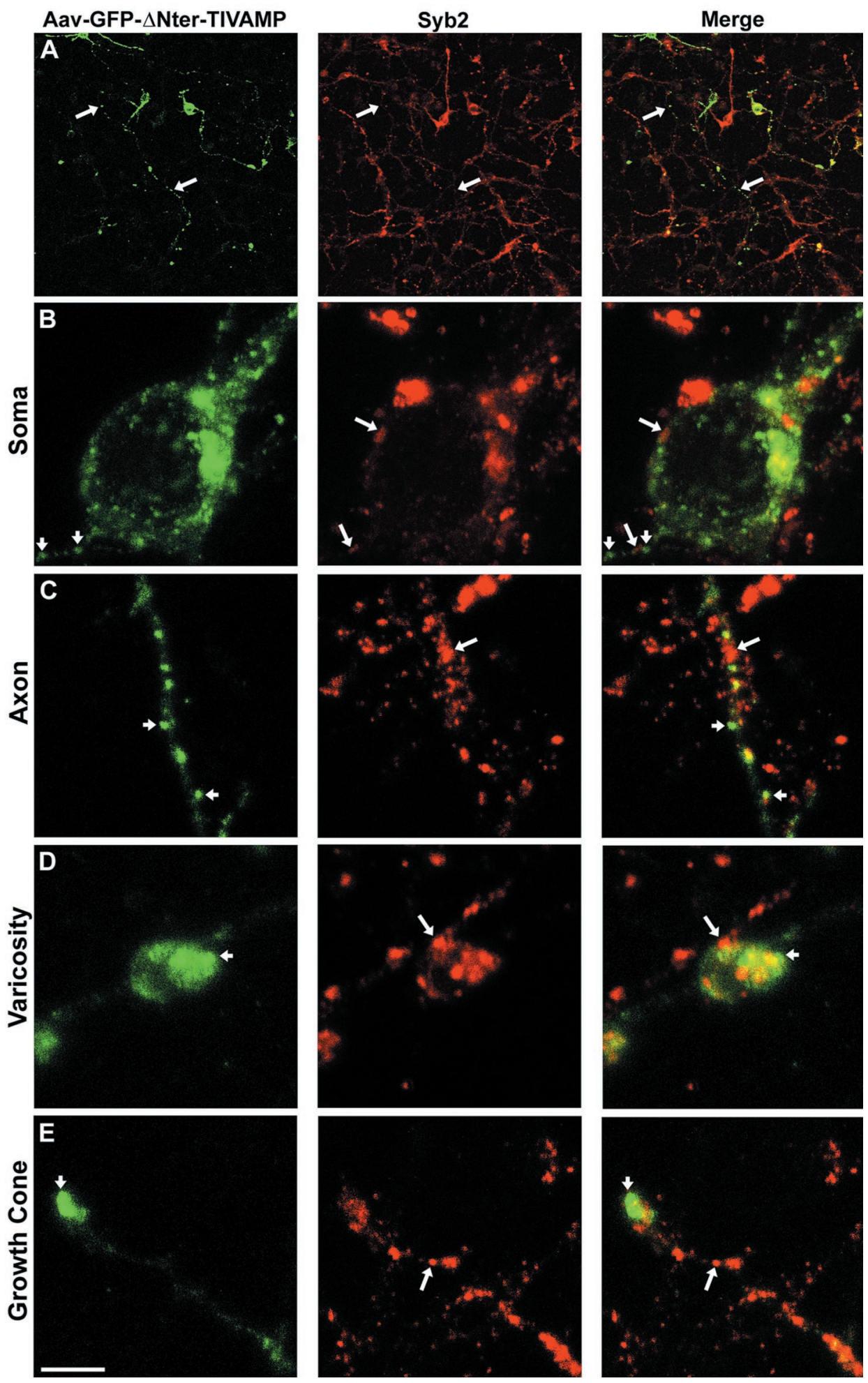

Figure 6. GFP- $\Delta$ Nter-TI-VAMP does not colocalize with synaptobrevin 2 . Rat embryonic neurons were infected with Aav carrying GFP- $\triangle$ Nter-TI-VAMP. After 6 div, the cells were fixed and permeabilized, incubated with a polyclonal antibody anti-GFP synaptobrevin 2 (Syb2), and observed by confocal microscopy. Low magnification images are shown in $A$. In all the other panels high magnification images of a cell body $(B)$, an axon $(C)$, a varicosity $(D)$, and a growth cone $(E)$, respectively, are shown. GFP$\Delta$ Nter-TI-VAMP (small arrows) does not colocalize with endogenous synaptobrevin 2 $(B-E$, large arrows $)$ in any of the different neuronal domains. A significant amount of GFP- $\Delta$ Nter-TI-VAMP was detected at the leading edge of the growth cone, in a region devoid of synaptobrevin 2. Scale bar: $A, 90$ $\mu \mathrm{m} ; B, C, E, 4.6 \mu \mathrm{m} ; D, 3 \mu \mathrm{m}$. a link between these two functions (Atwal et al., 2000). The relationship between cell geometry and regulation of the balance between cell growth and apoptosis has also been reported (Chen et al., 1997). Our results suggesting that the apoptosis observed after the expression of Nter-TI-VAMP is linked to the inhibition of axonal and dendritic outgrowth point to the interdependence between neuronal survival and neurite outgrowth.

The fact that expression of Nter-TI-VAMP blocked both axonal and dendritic outgrowths (Figs. 1, 3) indicates that both processes share common molecular mechanisms. As suggested by its localization at the leading edge of both axonal and dendritic growth cones, vesicles with fusion that is promoted by TI-VAMP could mediate exposure at the cell surface of proteins that are required both for axonal and dendritic outgrowth (Coco et al., 1999). The effect of Nter-TI-VAMP on the dendritic expression of EAAC1 (Fig. 2), a protein that may play a role in synaptogenesis (Coco et al., 1997), but not on the expression of GluR1, a protein of the mature dendrite (Eshhar et al., 1993; Richmond et 
al., 1996), suggests that TI-VAMP could be involved in exocytosis of a specific set of axonal and dendritic proteins that are expressed at early stages of neuronal development. Our observation that expression of $\triangle$ Nter-TI-VAMP had no effect on dendritic outgrowth can be explained if dendritic outgrowth cannot be further activated, at least under our conditions of culture. If this were so, the exocytosis mediated by TI-VAMP would be regulated differently in axons and dendrites. This would be in agreement with several experiments showing that dendritic and axonal outgrowths are controlled by different signals (Prochiantz, 1995). An alternative possibility is that TI-VAMP is primarily involved in axonal outgrowth and that dendritic outgrowth can proceed only when axonal outgrowth occurs normally. Indeed, our observations are reminiscent of recent work showing that amyloid precursor protein first appears in axons and is then transported to dendrites by transcytosis. Both amyloid precursor protein and TI-VAMP have been found in rafts (Bouillot et al., 1996; Lafont et al., 1999; Hooper et al., 2000) so the hypothesis that TI-VAMP would follow neuronal transcytosis is an appealing one. Our proposal that a common exocytotic mechanism mediates axonal and dendritic outgrowth at early stages of neuronal development is not contradictory with the high level of membrane trafficking polarity seen in mature neurons (Matteoli et al., 1995). In fact, we have previously shown that TI-VAMP concentrates in dendrites in mature hippocampal neurons and in the adult rat brain (Coco et al., 1999). Exocytosis mediated by TI-VAMP could participate in activity-dependent dendritic remodeling, a process reminiscent of early dendritic outgrowth (Maletic-Savatic et al., 1999; Toni et al., 1999). It will now be important to characterize the proteins that control the exocytotic pathway mediated by TI-VAMP. Specific axonal and dendritic factors are expected to regulate this pathway, thus accounting for differential control of the growth rate of axons and dendrites in different types of neurons, the result of a balance between exocytosis and endocytosis that may differ in axons and dendrites. Important factors involved in axonal and dendritic outgrowths may include rab proteins (Huber et al., 1995), GTPases of the Rac and Rho families (Nakayama et al., 2000), kinesins (Terada and Hirokawa, 2000), and regulators of endocytosis mediated by clathrin (Torre et al., 1994; Mundigl et al., 1998).

It has been shown that TI-VAMP is involved in several membrane trafficking steps in different cell types. It mediates apical exocytosis in epithelial cells (Galli et al., 1998; Lafont et al., 1999), degranulation in mast cells (Hibi et al., 2000), and participates in the EGF degradative pathway (Advani et al., 1999). This study establishes its intimate involvement in axonal and dendritic outgrowth. An appealing hypothesis could be that, among other cargo proteins, vesicles controlled by TI-VAMP could contain hydrolases. These enzymes could be involved in the processing of membrane proteins and/or they could fulfill a function once they are secreted. Secretion of certain hydrolases may be important for elongation of axons and pathfinding because they would allow for specific penetration of the extracellular medium by cleaving particular components of the basal lamina that would otherwise prevent elongation (McGuire and Seeds, 1990; Seeds et al., 1990). If this is the case, TI-VAMP-containing vesicles would be routed to different target membranes depending on the cell type: to endocytic structures in the case of fibroblasts or to plasma membranes in the case of epithelial cells, mast cells, and differentiating neurons. Such differences could also be correlated with different developmental stages. An alternative hypothesis would be that TI-VAMP is involved in transport from late endosomes to the plasma membrane and back and that this recycling mediates neurite outgrowth in developing neurons by a net exocytotic transport of proteins and lipids required for this process. The localization of TI-VAMP in adult dendrites (Coco et al., 1999) could suggest that this recycling is maintained in adult dendrites and respects a balance between exocytosis and endocytosis. Identification of the content of these vesicles in neurons is expected to yield proteins that are important for axonal outgrowth and may suggest new strategies for the treatment of severe traumatic nerve injuries.

\section{REFERENCES}

Advani RJ, Bae HR, Bock JB, Chao DS, Doung YC, Prekeris R, Yoo JS, Scheller RH (1998) Seven novel mammalian SNARE proteins localize to distinct membrane compartments. J Biol Chem 273:10317-10324.

Advani RJ, Yang B, Prekeris R, Lee KC, Klumperman J, Scheller RH (1999) VAMP-7 mediates vesicular transport from endosomes to lysosomes. J Cell Biol 146:765-775.

Atwal JK, Massie B, Miller FD, Kaplan DR (2000) The TrkB-Shc site signals neuronal survival and local axon growth via MEK and P13kinase. Neuron 27:265-277.

Bock JB, Scheller RH (1999) SNARE proteins mediate lipid bilayer fusion. Proc Natl Acad Sci USA 96:12227-12229.

Bock JB, Klumperman J, Davanger S, Scheller RH (1997) Syntaxin 6 functions in trans-Golgi network vesicle trafficking. Mol Biol Cell 8:1261-1271.

Bouillot C, Prochiantz A, Rougon G, Allinquant B (1996) Axonal amyloid precursor protein expressed by neurons in vitro is present in a membrane fraction with caveolae-like properties. J Biol Chem 271:7640-7644.

Bradke F, Dotti CG (1997) Neuronal polarity: vectorial cytoplasmic flow precedes axon formation. Neuron 19:1175-1186.

Bradke F, Dotti CG (2000a) Differentiated neurons retain the capacity to generate axons from dendrites. Curr Biol 10:1467-1470.

Bradke F, Dotti CG (2000b) Establishment of neuronal polarity: lessons from cultured hippocampal neurons. Curr Opin Neurobiol 10:574-581.

Chen CS, Mrksich M, Huang S, Whitesides GM, Ingber DE (1997) Geometric control of cell life and death. Science 276:1425-1428.

Coco S, Verderio C, Trotti D, Rothstein JD, Volterra A, Matteoli M (1997) Non-synaptic localization of the glutamate transporter EAAC1 in cultured hippocampal neurons. Eur J Neurosci 9:1902-1910.

Coco S, Raposo G, Martinez S, Fontaine JJ, Takamori S, Zahraoui A, Jahn R, Matteoli M, Louvard D, Galli T (1999) Subcellular localization of tetanus neurotoxin-insensitive vesicle-associated membrane protein (VAMP)/VAMP7 in neuronal cells: evidence for a novel membrane compartment. J Neurosci 19:9803-9812.

Craig AM, Wyborski RJ, Banker G (1995) Preferential addition of newly synthesized membrane protein at axonal growth cones. Nature 375:592-594

D’Esposito M, Ciccodicola A, Gianfrancesco F, Esposito T, Flagiello L, Mazzarella R, Schlessinger D, D'Urso M (1996) A synaptobrevin-like gene in the Xq28 pseudoautosomal region undergoes $\mathrm{X}$ inactivation. Nat Genet 13:227-229.

Dotti CG, Banker GA (1987) Experimentally induced alteration in the polarity of developing neurons. Nature 330(6145):254-256.

Dotti CG, Sullivan CA, Banker GA (1988) The establishment of polarity by hippocampal neurons in culture. J Neurosci 8:1454-1468.

Du B, Wu P, Boldt-Houle DM, Terwilliger EF (1996) Efficient transduction of human neurons with an adeno-associated virus vector. Gene Ther 3:254-261.

Eshhar N, Petralia RS, Winters CA, Niedzielski AS, Wenthold RJ (1993) The segregation and expression of glutamate receptor subunits in cultured hippocampal neurons. Neuroscience 57:943-964.

Futerman AH, Banker GA (1996) The economics of neurite outgrowththe addition of new membrane to growing axons. Trends Neurosci 19:144-149.

Galli T, Garcia EP, Mundigl O, Chilcote TJ, DeCamilli P (1995) v- and t-SNAREs in neuronal exocytosis: a need for additional components to define sites of release. Neuropharmacology 34:1351-1360.

Galli T, Zahraoui A, Vaidyanathan VV, Raposo G, Tian JM, Karin M, Niemann H, Louvard D (1998) A novel tetanus neurotoxin-insensitive vesicle-associated membrane protein in SNARE complexes of the apical plasma membrane of epithelial cells. Mol Biol Cell 9:1437-1448.

Garcia EP, McPherson PS, Chilcote TJ, Takei K, De Camilli P (1995) rbSec1A and B colocalize with syntaxin 1 and SNAP-25 throughout the axon, but are not in a stable complex with syntaxin. J Cell Biol 129:105-120.

Grosse G, Grosse J, Tapp R, Kuchinke J, Gorsleben M, Fetter I, HohneZell B, Gratzl M, Bergmann M (1999) SNAP-25 requirement for dendritic growth of hippocampal neurons. J Neurosci Res 56:539-546. 
Hibi T, Hirashima N, Nakanishi M (2000) Rat basophilic leukemia cells express syntaxin-3 and VAMP-7 in granule membranes. Biochem Biophys Res Commun 271:36-41.

Hooper NM, Trew AJ, Parkin ET, Turner AJ (2000) The role of proteolysis in Alzheimer's disease. Adv Exp Med Biol 477:379-390.

Huber LA, Dupree P, Dotti CG (1995) A deficiency of the small GTPase rab8 inhibits membrane traffic in developing neurons. Mol Cell Biol 15:918-924.

Igarashi M, Kozaki S, Terakawa S, Kawano S, Ide C, Komiya Y (1996) Growth cone collapse and inhibition of neurite growth by Botulinum neurotoxin C1: A t-SNARE is involved in axonal growth. J Cell Biol 134:205-215.

Jahn R, Sudhof TC (1999) Membrane fusion and exocytosis. Annu Rev Biochem 68:863-911.

Johannes L, Galli T (1998) Exocytosis: SNAREs drum up! Eur J Neurosci 10:415-422.

Lafont F, Verkade P, Galli T, Wimmer C, Louvard D, Simons K (1999) Raft association of SNAP receptors acting in apical trafficking in Madin-Darby canine kidney cells. Proc Nat Acad Sci USA 96:3734-3738.

Mainguy G, Luz Montesinos M, Lesaffre B, Zevnik B, Karasawa M, Kothary R, Wurst W, Prochiantz A, Volovitch M (2000) An induction gene trap for identifying a homeoprotein-regulated locus. Nat Biotechnol 18:746-749.

Maletic-Savatic M, Malinow R, Svoboda K (1999) Rapid dendritic morphogenesis in CA1 hippocampal dendrites induced by synaptic activity. Science 283:1923-1927.

Maniatis T, Fritsch EF, Sambrook J (1982) Molecular cloning: a laboratory manual, Ch 16, pp 33-36. Cold Spring Harbor, NY: Cold Spring Harbor Laboratory.

Martinez-Arca S, Alberts P, Zahraoui A, Louvard D, Galli T (2000a) Role of tetanus neurotoxin insensitive vesicle-associated membrane protein (TI-VAMP) in vesicular transport mediating neurite outgrowth. J Cell Biol 149:889-899.

Martinez-Arca S, Alberts P, Galli T (2000b) Clostridial neurotoxininsensitive vesicular SNAREs in exocytosis and endocytosis. Biol Cell 92:449-453.

Matteoli M, Verderio C, Krawzeski K, Mundigl O, Coco S, Fumagalli G, DeCamilli P (1995) Mechanisms of synaptogenesis in hippocampal neurons in primary culture. J Physiol (Paris) 89:51-55.

McGuire PG, Seeds NW (1990) Degradation of underlying extracellular matrix by sensory neurons during neurite outgrowth. Neuron 4:633-642

Miyasaka N, Arimatsu Y, Takiguchihayashi K (1999) Foreign gene expression in an organotypic culture of cortical anlage after in vivo electroporation. NeuroReport 10:2319-2323.

Mundigl O, Ochoa GC, David C, Slepnev VI, Kabanov A, DeCamilli P (1998) Amphiphysin I antisense oligonucleotides inhibit neurite outgrowth in cultured hippocampal neurons. J Neurosci 18:93-103.

Nakamura N, Lowe M, Levine TP, Rabouille C, Warren G (1997) The vesicle docking protein p115 binds GM130, a cis-Golgi matrix protein, in a mitotically regulated manner. Cell 89:445-455.
Nakayama AY, Harms MB, Luo L (2000) Small GTPases Rac and Rho in the maintenance of dendritic spines and branches in hippocampal pyramidal neurons. J Neurosci 20:5329-5338.

Osen-Sand A, Staple JK, Naldi E, Schiavo G, Rossetto O, Petitpierre S, Malgaroli A, Montecucco C, Catsicas S (1996) Common and distinct fusion proteins in axonal growth and transmitter release. J Comp Neurol 367:222-234.

Patil AR, Thomas CJ, Surolia A (2000) Kinetics and the mechanism of interaction of the endoplasmic reticulum chaperone, calreticulin, with monoglucosylated (Glc1Man9GlcNAc2) substrate. J Biol Chem 275:24348-24356.

Polverino AJ, Patterson SD (1997) Selective activation of caspases during apoptotic induction in HL-60 cells. Effects of a tetrapeptide inhibitor. J Biol Chem 272:7013-7021.

Prochiantz A (1995) Neuronal polarity: giving neurons heads and tails. Neuron 15:743-746.

Richmond SA, Irving AJ, Molnar E, McIlhinney RA, Michelangeli F, Henley JM, Collingridge GL (1996) Localization of the glutamate receptor subunit GluR1 on the surface of living and within cultured hippocampal neurons. Neuroscience 75:69-82.

Rousselet A, Autillo-Touati A, Araud D, Prochiantz A (1990) In vitro regulation of neuronal morphogenesis and polarity by astrocytederived factors. Dev Biol 137:33-45.

Salvetti A, Oreve S, Chadeuf G, Favre D, Cherel Y, Champion-Arnaud P, David-Ameline J, Moullier P (1998) Factors influencing recombinant adeno-associated virus production. Hum Gene Ther 9:695-706.

Seeds NW, Haffke S, Christensen K, Schoonmaker J (1990) Cerebellar granule cell migration involves proteolysis. Adv Exp Med Biol 265:169-178.

Slack RS, Miller FD (1996) Viral vectors for modulating gene expression in neurons. Curr Opin Neurobiol 6:576-583.

Söllner T, Whiteheart SW, Brunner M, Erdjument-Bromage H, Geromanos S, Tempst P, Rothman JE (1993) SNAP receptors implicated in vesicle targeting and fusion. Nature 362:318-324.

Terada S, Hirokawa N (2000) Moving on to the cargo problem of microtubule-dependent motors in neurons. Curr Opin Neurobiol 10:566-573.

Toni N, Buchs PA, Nikonenko I, Bron CR, Muller D (1999) LTP promotes formation of multiple spine synapses between a single axon terminal and a dendrite. Nature 402:421-425.

Torre E, McNiven MA, Urrutia R (1994) Dynamin 1 antisense oligonucleotide treatment prevents neurite formation in cultured hippocampal neurons. J Biol Chem 269:32411-32417.

Wong SH, Xu Y, Zhang T, Hong W (1998) Syntaxin 7, a novel syntaxin member associated with the early endosomal compartment. J Biol Chem 273:375-380.

Xiao X, Li J, Samulski RJ (1998) Production of high-titer recombinant adeno-associated virus vectors in the absence of helper adenovirus. J Virol 72:2224-2232.

Zakharenko S, Popov S (1998) Dynamics of axonal microtubules regulate the topology of new membrane insertion into the growing neurites. J Cell Biol 143:1077-1086. 